\title{
DYNAMIC BEAM-BEAM EFFECTS MEASURED AT KEKB
}

\author{
T. Ieiri", Y. Funakoshi, T. Kawamoto, M. Masuzawa, and M. Tobiyama \\ KEK, Oho1-1, Tsukuba, Ibaraki 305-0801, Japan
}

\section{Abstract}

A series of measurements were carried out to investigate the dynamic beam-beam effects. The beambeam kick and the beam-beam tune-shift were obtained by comparing the beam parameters between a colliding bunch and a non-colliding one. The horizontal beam size at the interaction point estimated from a beam-beam kick curve slightly decreased as the beam-beam parameter increased. The horizontal emittance estimated from the beam-beam tune shift was larger than an unperturbed emittance and agreed with calculated emittance using a higher-mode tune. A ratio of the beta function between two locations in the ring, however, showed a big disparity between measured values and a calculation.

\section{INTRODUCTION}

KEKB [1] is a multi-bunch, high-current, electron/positron collider for $B$-meson physics. The collider consists of two storage rings: the Low Energy Ring (LER) for a $3.5-\mathrm{GeV}$ positron beam and the High Energy Ring (HER) for $8-\mathrm{GeV}$ electrons. Both rings store more than 1,200 bunches, where the harmonic number is 5120 with an RF frequency of $509 \mathrm{MHz}$. The two beams collide at one interaction point (IP) with a horizontal crossing angle of $22 \mathrm{mrad}$. Unlike conventional singlering colliders, the beam parameters are different between the two rings, which makes collisions complicate. The maximum luminosity of $1.5 \times 10^{34} \mathrm{~cm}^{-2} \mathrm{sec}^{-1}$ has been achieved so far, which is the best in the world.

The dynamic beam-beam effects at KEKB are remarkable, since the betatron tunes are set close to a half integer in addition to a high beam-beam parameter. The beam parameters vary, depending on the beam intensity and the beam size. It is important to obtain experimental evidence of the dynamic effects from a viewpoint of beam dynamics.

In order to investigate any dynamic beam-beam effects, the beam parameters have been measured by comparing them between collision and non-collision bunches [2]. We have found that collisions occur with a horizontal orbit offset to obtain the maximum luminosity and that the offset is related to an effect of the electron cloud which might play an important role in the beam-beam interaction [3].

\section{BEAM-BEAM EFFECTS}

The beam-beam collision is supposed to install nonlinear quadrupole field. The additional field distorts the optical parameters around the rings. The beta beat at a phase advance of $\Delta \varphi_{s}$ from the IP is given by

\#) Email: takao.ieiri@kek.jp

$$
\Delta \beta(s)=\frac{\beta(s) \beta^{*}}{2 \sin (2 \pi v)} \Delta k^{*} \cos \left(2 \pi v-2 \mid \Delta \varphi_{s}\right),
$$

where $\Delta k^{*}$ is a field gradient due to the beam-beam force and $v$ is the betatron tune, $\beta(s)$ and $\beta^{*}$ are the beta function at a phase advance of $\Delta \varphi_{s}$ and at the IP, respectively. The beam-beam collision also changes the emittance of each beam. The beta function at the IP decreases and the emittance increases as the beam-beam parameter increases. Thus the horizontal beam size at the IP slightly reduces, compared with a non-perturbed beam size. These dynamical effects should affect the luminosity performance.

When two beams collide with a position offset at the IP, they are kicked by the space charge of the opposite beam and an orbit of each beam is distorted around the ring. A position monitor located at a phase advance of $\Delta \varphi_{\mathrm{d}}$ from the IP detects an orbit change due to collisions. The position shift at a detector is given by

$$
\Delta X_{\text {det. }}=\frac{\sqrt{\beta_{\text {det } .} \beta_{x}^{*}}}{2 \sin (\pi v)} \theta_{b-b} \cos \left(\pi v-\left|\Delta \varphi_{\mathrm{d}}\right|\right) .
$$

Here, $\beta_{\text {det. }}$ is the beta functions at a detector and $\theta_{b-b}$ is the beam-beam kick angle. Figure 1 shows the horizontal beam-beam kick, based on the rigid Gaussian model, as a function of the horizontal offset with a parameter of the effective beam size defined by $\Sigma_{x}=\sqrt{\left(\sigma_{x}^{+}\right)^{2}+\left(\sigma_{x}^{-}\right)^{2}}$. The superscript \pm denotes positron and electron bunches. We can estimate the effective beam size at the IP from the beam-beam kick curve. Around zero-offset, the horizontal beam-beam kick is inversely proportional to square of the effective horizontal size for a flat beam. The calculated horizontal sizes without the dynamic effect are $\sigma_{x}^{+}=103 \mu \mathrm{m}$ and $\sigma_{x}^{-}=116 \mu \mathrm{m}$ at the IP, which results in an effective horizontal size of $\Sigma_{\mathrm{x}}=155 \mu \mathrm{m}$.

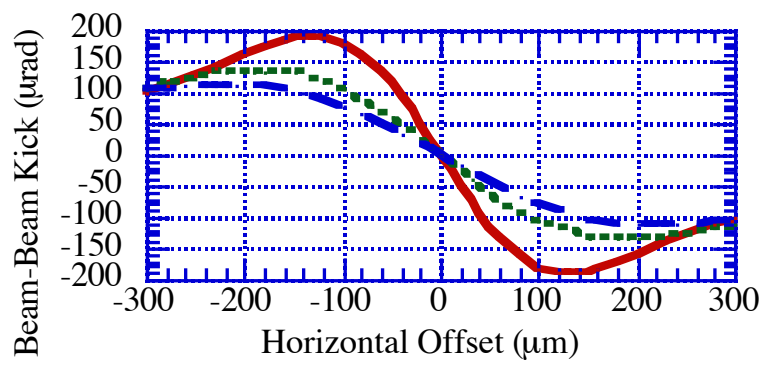

Figure 1: Beam-beam kicks as a function of the horizontal offset, assuming the effective horizontal beam sizes of $100 \mu \mathrm{m}$ in red solid line, $141 \mu \mathrm{m}$ in green dotted line and $170 \mu \mathrm{m}$ in blue broken line, and the effective beam size is $2.83 \mu \mathrm{m}$ vertically for all cases under the same bunch current. 
When the position shift is measured at two locations with the phase advance of $\Delta \varphi_{1}$ and $\Delta \varphi_{2}$ in the ring, a ratio of two position-shifts is given by

$$
\frac{\Delta X_{2}}{\Delta X_{1}}=\sqrt{\frac{\beta_{2}}{\beta_{1}}} \cdot \frac{\cos \left(\pi v-\left|\Delta \varphi_{2}\right|\right)}{\cos \left(\pi v-\left|\Delta \varphi_{1}\right|\right)} .
$$

The position ratio is independent of the beam-beam-kick and gives a ratio of the beta function including a phase advance at two locations. We might obtain information about the dynamic beta from the ratio of the position shift.

On the other hand, the beam-beam interaction produces a new set of two betatron tunes by mixing two unperturbed tunes. We call the two perturbed modes the higher $(H-)$ mode and the lower $(L-)$ mode, unlike the $\Sigma$ (or zero) and the $\pi$ - modes in a single-ring collider. The $H$-mode tune increases, however, the $L$-mode tune is almost constant, as a function of the coherent beam-beam parameter, as shown in Fig. 2. The coherent beam-beam parameter is just half of the incoherent beam-beam parameter, $\xi_{q}$ in the rigid Gaussian model; when both of the sizes are equal, it is $\xi_{q} \approx \Xi_{q}^{+}+\Xi_{q}^{-}$.

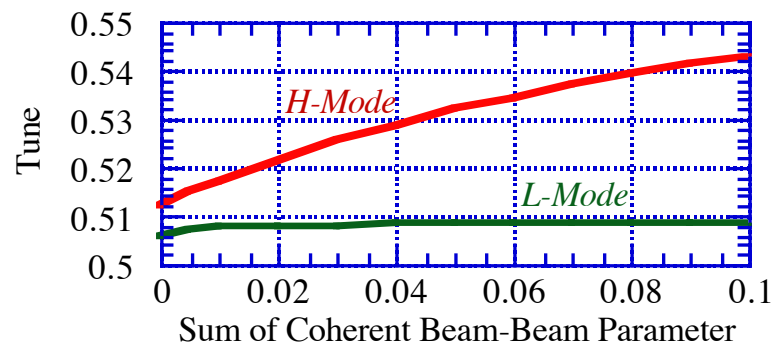

Figure 2: $H$-mode tune and $L$-mode tune as a function of sum of the coherent beam-beam parameter, where unperturbed fractional tunes are 0.506 and 0.512 .

The relation between the horizontal emittance and the coherent beam-beam parameter is expressed by

$$
\varepsilon_{x}^{+}+\varepsilon_{x}^{-}=\frac{r_{e}}{2 \pi} \cdot\left(\frac{N^{+}}{\gamma^{-}}+\frac{N^{-}}{\gamma^{+}}\right) \cdot \frac{1}{\left(\Xi_{x}^{+}+\Xi_{x}^{-}\right)} .
$$

Here, $\gamma$ is the relativistic factor, $r_{e}$ the classical electron radius, $N$ the number of particles in a bunch and $\beta_{x}^{+}=\beta_{x}^{-}$is assumed. We can estimate the sum of the horizontal emittance from the coherent tune shift. The calculated emittance without the dynamic effect is $\varepsilon_{x}^{+}=18 \mathrm{nmrad}$ and $\varepsilon_{x}^{-}=24 \mathrm{nmrad}$. The sum emittance is 42 nmrad.

\section{MEASUREMENT CONDITION}

Two sets of gated beam-position monitors are installed in each ring. Table 1 gives the optical parameters at the monitors without the dynamic effects. One of the monitors, LER-2, is located at an optimum phase advance from the IP with a high horizontal beta function of $73 \mathrm{~m}$ and has the highest sensitivity for detecting a horizontal beam-beam kick. The other monitors have about half sensitivity. The beta function and the phase advance, however, might change due to the dynamic effects. A calculation shows that the beta function at the IP decreases, on the other hand, that at the LER-2 increases as the beam-beam parameter increases. It was found that the product of the horizontal beta functions at the IP and at the LER-2 was almost constant, regardless of the beambeam parameter. Therefore, the horizontal position shift at the LER-2 is proportional to the beam-beam kick.

Table 1 Horizontal phase advance from the IP and the beta function at the detectors without the dynamical effects. Sensitivity for measuring a horizontal position shift is relative values, when we set that of the LER-2 to be 1.0 as a standard.

\begin{tabular}{|c|c|c|c|c|}
\hline & $\begin{array}{c}\text { LER-1 } \\
\text { BPM\#098 }\end{array}$ & $\begin{array}{c}\text { LER-2 } \\
\text { BPM\#104 }\end{array}$ & $\begin{array}{c}\text { HER-1 } \\
\text { BPM\#097 }\end{array}$ & $\begin{array}{c}\text { HER-2 } \\
\text { BPM\#100 }\end{array}$ \\
\hline$\left|\Delta \phi_{x}\right| / 2 \pi$ & 9.34 & 9.77 & 35.35 & 35.12 \\
\hline$\beta_{x}(\mathrm{~m})$ & 39.29 & 73.14 & 21.14 & 31.89 \\
\hline Sensitivity & -0.63 & 1.00 & 0.45 & 0.42 \\
\hline
\end{tabular}

\section{RESULTS}

\section{Horizontal beam size at the IP}

The measurement was carried out with changing the beam orbit of the electron beam horizontally at the IP, while the orbit of the positron beam and the vertical orbit of both beams were kept constant. The horizontal beambeam kick was measured using the LER-2 detector and calculated using the rigid Gaussian model, as shown in Fig. 3. We can estimate the effective beam size from the slope around zero-kick and the offset value providing the maximum kick. Assuming both beam sizes are equal, the beam-beam kick with a low current is fitted with an rms size of $\sigma_{\mathrm{x}}^{ \pm}=110 \mu \mathrm{m}$. The maximum deflection occurs at an offset of about $204 \mu \mathrm{m}$, which corresponds to an effective size of $\Sigma_{\mathrm{x}}=155 \mu \mathrm{m}$. Similar measurements were carried out with some different bunch currents. Figure 4 shows fitted and calculated effective sizes as a function of the beam-beam parameter obtained from the tune shift measurement. The fitted sizes were obtained from the slope around zero-offset in the beam-beam kick. The fitted effective beam size slightly decreased as the beambeam parameter increased. The measurement is consistent with prediction based on the dynamic effects.

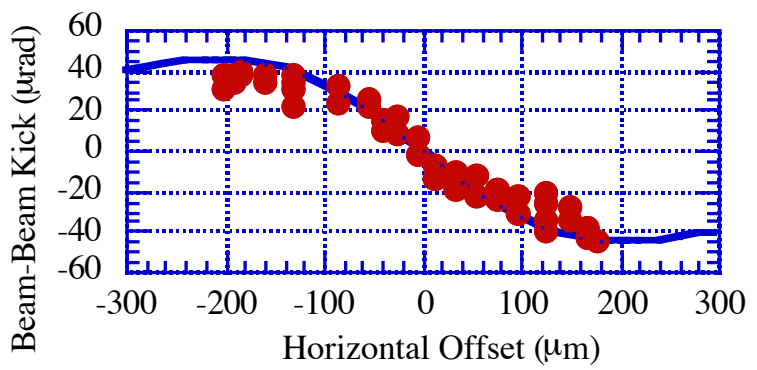

Figure 3: Dots are the beam-beam kick measured at a low current as a function of horizontal offset. Solid lines are calculated beam-beam kick, assuming both horizontal 
beam sizes are equal to $110 \mu \mathrm{m}$ and a vertical size to 2 $\mu \mathrm{m}$.

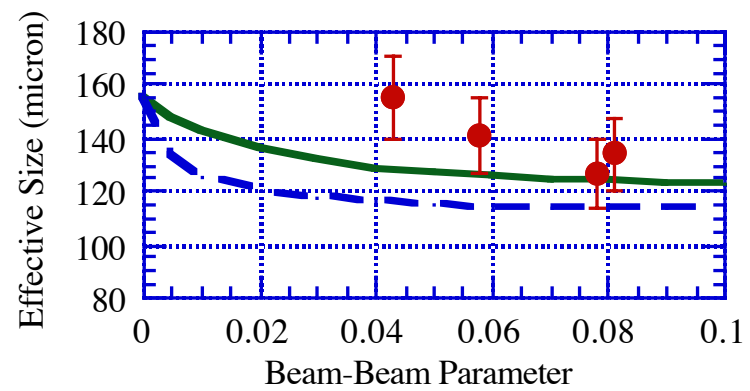

Figure 4: The effective horizontal beam sizes, dots are fitted sizes and lines are calculated values with fractional tunes of 0.540 (green solid line) and 0.510 (blue broken line), as a function of the beam-beam parameter. The tunes in the measurement were around 0.510 for the $L$ mode and 0.538 to 0.549 for the $H$-mode.

\section{Ratio of the beta function}

The position shift was measured at two locations in the LER and the HER while changing the horizontal offset or the beam-beam kick at the IP. Figure 5 shows the measured ratio of the position shift at three different currents. A calculated ratio is also shown, where the beta function and the phase advance from the IP to the detector were obtained using the rigid Gaussian model with two different tunes. Comparing the measurement with the calculation, there is a big disparity of about $30 \%$. Similar measurement was carried out with the detectors of HER-1 and LER-2. The measurement also did not agree with calculation. However, the position sensitivity in a single beam agreed with expectation.

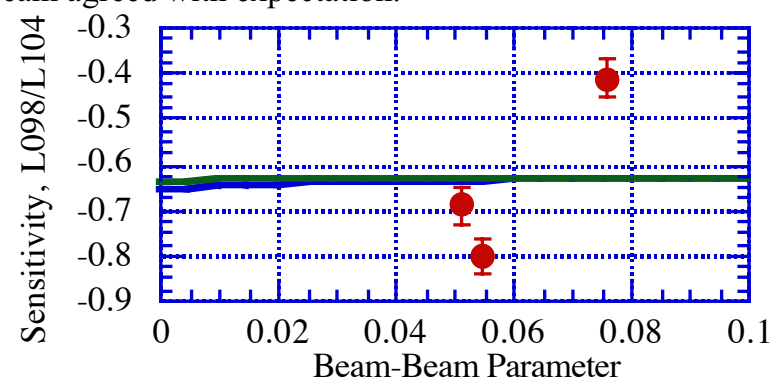

Figure 5: The ratio of the position shift between two BPMs of LER-1 and LER-2 as a function of the beambeam parameter. Solid lines are a calculated position ratio using fractional tunes of 0.510 and 0.540 and dots are measured ones.

\section{Dynamic emittance}

The horizontal emittance can be estimated from the coherent tune shift as given in Eq. (4). Figure 6 shows the sum of the emittance estimated from the tune shift as a function of a normalized intensity, $N^{+} / \gamma^{-}+N^{-} / \gamma^{+}$. We find that the measured sum emittance increases with increasing the beam intensity. Figure 6 also shows two curves of calculated emittance, since two modes of tune exist: $H$-mode and $L$-mode, two values of emittance are obtained. The calculated sum emittance without the dynamic effect is $42 \mathrm{nmrad}$. The estimated emittance from the tune shift agrees with the calculated emittance using not $L$-mode tune, but $H$-mode tune.

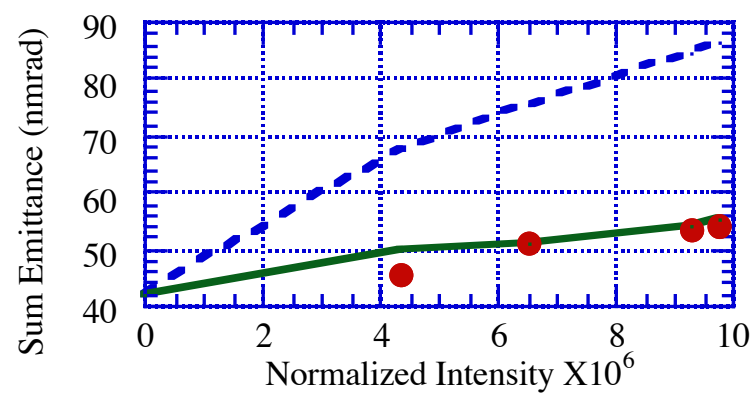

Figure 6: Sum of the emittance estimated from the tune shift (dots) and calculated sum emittance with horizontal tunes of 0.512 (blue broken line) and of 0.540 (green solid line) as a function of the normalized intensity.

\section{DISCUSSION}

It was demonstrated that the measured horizontal beam size at the IP decreased as the beam-beam parameter increased and was slightly smaller than an unperturbed size at a high beam-beam parameter. The measurement is consistent with expectation based on the dynamic effects. However, we cannot determine which tune is useful in Fig. 4, because of poor accuracy in the measurement. More accurate measurement is required.

There was a big disparity in the beta function including a phase advance between the measurement and our calculation based on the rigid Gaussian model. It is not clear whether there is a big error in the measurement or the actual beta function is seriously distorted. In order to solve the disparity, the beta function should be measured by another method.

The sum emittance obtained using a Yokoya factor [4] of 1.31 was slightly higher than an unperturbed one. The experimental result agrees with the emittance calculated using $H$-mode tune, not $L$-mode.

The authors would like to thank the members of the KEKB commissioning group for their help. This work was partially supported by Grant-in-Aid Scientific Research (16540271) from Japan Society for the Promotion of Science and Technology.

\section{REFERENCES}

[1] K. Akai et al., Nucl. Instrum. Methods A499 191 (2003).

[2] T. Ieiri et al., Proc. of SAST'03, Tsukuba, Japan, 446 (2003).

[3] T. Ieiri et al., Proc. of EPAC'04, Lucerne, Switzerland, 2095 (2004).

[4] K. Yokoya and H. Koiso, Particle Accelerator, 27181 (1991). 